\title{
Analysis of the Temporal and Geographical Distribution of a Chinese Learning Website Visitors
}

\author{
Xiaochen Li and Yan Xu
}

\begin{abstract}
As China developing in a rapid step, there is a tremendous growing in the foreigners' interest of learning Chinese and exploring the Chinese culture. Along with this, more and more foreigners choose the Internet as an access to Chinese learning resources. In this paper we process an important Chinese learning website's Web logs and analyze the users' distribution. Based on deep statistics and analysis, we propose several conclusions about the temporal and geographical distribution characteristics of the Chinese learning website visitors. For the temporal distribution, we observe an obvious pattern: (i) it's a cyclic pattern with a period of one week, (ii)Thursday holds the peak of user numbers within a specific week, (iii) there is a downward trend of the user numbers per day during weekdays. For the geographical distribution of the users, we present experiments on two levels: (a) countries and regions, (b) continents. Understanding the temporal and geographical distribution characteristics of Chinese learning website visitors brings us a better knowledge of the users' preference and gives us pointers for research, so that we can improve the Chinese learning website and then attain a better adaption of the users' habits and need.
\end{abstract}

Index Terms - Chinese learning, website usage, distribution of website visitors

\section{INTRODUCTION AND BACKGROUND}

Recently Chinese language learning and Chinese culture is gaining interest from an increasing number of people around the world. And the prevailing usage of the Internet provides a convenient way for users to access on-line Chinese learning resources. Thus, understanding who are deploying the Chinese learning website and when they visit the website becomes an important research topic. Because these will not only provide us clues which can help to improve the architecture of the website and the Multilanguage service of pages, but also give pointers to the adjustments of the number of servers and the bandwidth. Based on these improvements and adjustments, we can attain a balance between a better online service and a more rational use of resources.

Cooley et al. [1] proposed that how a website is used is an important input to Fig out the complexity of tasks such as website architecture and web server architecture. Web Usage

Manuspcript received September 20, 2012; revised December 16, 2012 This work was supported in part by the Chinese National Undergraduate Research and Innovation Plan, National Natural Science Foundation of Beijing under Grant 4122076 and Education science Foundation of Beijing under Grant AHA09110.

The authors are with the College of Information Science, Beijing Language and Culture University, Beijing, 100083 China (e-mail: xcli@outlook.com, xuy@blcu.edu.cn).
Mining is the application of data mining techniques to large Web data repositories in order to produce results that can be used in the design tasks mentioned above. Web logs play a significant role in the web mining process because Web logs are crucial information sources for analyzing users' action and these information sources are issued by users themselves.

In this paper, we process and analyze the Web logs of a Chinese learning website. In Section II we discuss previous work on Web logs mining, research on website usage and Web logs and data visualization. We design several algorithms to identify users from these logs and propose some statistics of the users (see Section III). This processed data provides us information of the website users' distribution from two dimensions (see Section IV ): the temporal dimension and the geographical dimension. Finally, we conclude and discuss further observations in Section V.

The paper makes the following contributions: (1) we Fig out the periodical pattern of the change in the user number, (2) we describe the users' geographical distribution on the country level and the continent level. To the best of our knowledge, our study is the first that provides a detailed users' temporal and geographical distribution of a Chinese learning website, which is a hot issue of education. Learning about users' distribution characteristics makes significant difference because it leads to new research directions. Moreover, it helps us gain a better knowledge of website users and thus help us improve the website organization in order to provide better online services.

\section{RELATED WORK}

Analysis of the Chinese learning website visitors' temporal and geographical distribution is a new research branch so that there are rare related articles. But we can still learn from some research methods.

\section{A. Information Objects}

1) Clicks.

Generally, there are several links in a specific page. We define a click as a user clicks on one of these links and enter a new page.

2) Users.

Web server logs provide limited information about users. As a result, we can only get a user's IP address and his identification information (User Agent). However, users' accesses to network vary widely for their using caching, proxy servers and firewalls. All of these applications bring lots of troubles and complexities to the identification of users. For instance, when different users access to the server with a same proxy server, Web server logs will show the same IP address corresponding to these different users. Another 
instance is that sometimes a specific user will use different web browsers on his computer when accessing to the server. In this case, the Web server logs cannot distinguish clearly whether these logs belong to the same user or belong to different users. In Section III we will talk about algorithms designed to identify users in our paper.

\section{3) Sessions.}

Identification of users is often followed by the detection of sessions. The so-called session refers to a chronological set which consists of several pages visited by a specific user. By analyzing the timing set, we can gain some knowledge of users' interest and preferences. As a result, the detection of sessions is a key step in Web log mining and various algorithms have been proposed in order to get a better performance.

For instance, the timeout method for sessions or pages with its key point of fixing the maximum time (cutoff value) of the session or the time spent on a specific page by a user, is relatively easier to implement. According to Goker [2], the best time threshold value for session is 25.5 minutes. The cutoff value is dependent on the goal of specific analysis. Most of the commercial products set their cutoff value as 30 minutes. In this paper, we also choose 30 minutes as our cutoff value of sessions. The timeout method for pages takes users' habits into consideration when compared with the timeout method for sessions. This results in a better performance.

However, in timeout method the cutoff value is simply set without deeply analysis of the website's contents and the users' habits. As a result, the method has its limitations to some extent. Cooley et al. [1] proposed a transaction method to identify sessions. The main idea of this method is that whether users stay on a specific page and the time spent on this page are dependent on the category this page belongs to, namely, the page is a content page or a secondary page. Although this method results in a more precise performance, it still has some drawbacks: the method itself is complex to implement because we should pre-judge the category of the page. In addition, the formation of each session contains only one content page. As a result, usage of this method is not as wide as that of the timeout method.

\section{B. Research on Website Usage and Web Logs}

There has been lots of research which focus on several aspects on website usage. Mahlke [3] and Hans [4] discussed factors influencing the usage of websites, including perceived usefulness, ease of use, hedonic quality and visual attractiveness etc. Hans [5] also proposed a technology acceptance model to predict the website usage. Cohen [6] developed a two-tiered model for analyzing library website usage statistics.

Numerous of analysis has also been proposed on Web logs. Beitzel's study focused on investigating the nature of changes in the query stream of a very large search service over. He also proposed a method of temporal log analysis that can be used to study changing topical trends over time in addition to the traditional static analysis [7]. Silverstein et al. found that for $85 \%$ of the queries in Alta Vista only the first result screen is viewed, and $77 \%$ of the sessions contain only 1 query [8].
Domain-specific search behavior has been studied in the context of searching for people on the web [9], biomedical information seeking [10], blog search [11], and so on. Weerkamp et al. [9] analyzed a large-scale log of people search on the web, providing detailed statistics at the level of queries, sessions and users. Lin et al. [10] examined the relationship between initial search results and the overall utility of interaction, finding that similarity-based browsing tool helps in the case of poor initial retrieval results. Mishne et al. [11] focused on the analysis of blog search behavior, finding that blog searches typically include named entities of interests [12]. In our paper, we focus on the Chinese learning website.

\section{Data Visualization}

Data visualization is the study of the visual representation of data, meaning "information which has been abstracted in some schematic form, including attributes or variables for the units of information" [13]. Several tools and technologies have been developed to achieve a balance between aesthetic form and functionality need, such as tables, histograms and bar graphs. Another tool used in our paper for data visualization is data map, which means displaying data on a geographical map to provide an insight to the knowledge of geographical distribution in data. There is a variety of researches on the data map. For instance, Healey [14] described a technique for choosing multiple colors for use during data visualization.

\section{Chinese LEARning Website AND DATA Processing}

\section{A. Web Logs and Data Cleaning}

The main data source of this paper is the Web logs issued by Beijing Language and Culture University Website server. BLCU is the only international university in China with its main task set at teaching Chinese language and culture to foreign students. There are several centers for studying Chinese on campus and the university also provides a bunch of online Chinese learning resources for foreigners. The Web $\log$ data was collected between July 1, 2011 and August 31, 2011. During this period there was no major update or change to Beijing Language and Culture University's website which guarantees the data's comparability.

In many computer science fields, one needs to prepare quality data by pre-processing the raw data. In practice, it has been generally found that data cleaning and preparation takes approximately $80 \%$ of the total data engineering effort. Data preparation is, therefore, a crucial research process. First, BLCU's website (www.blcu.edu.cn) consists of several department-level sub-websites and Chinese learning centers' websites. With the focus of analyzing behaviors of Chinese learners, we process the Web logs and eliminate records irrelevant to Chinese learning. Second, elimination of the items deemed irrelevant can be reasonably accomplished by checking the suffix of the URL name. For instance, all log entries with filename suffixes such as gif, GIF, jpeg, JPEG, jpg, JPG and map can be removed [1]. Log cleaning algorithm used in this article is presented as follows:

Step 1 Remove the Web log entries which are irrelevant to 
Chinese learning resources.

Step 2 Remove the Web log entries whose request-status is not 200.

Step 3 Remove the log entries whose suffixes of the URL belong to a defined $\operatorname{set}^{1}$.

The data size of initial Web logs is on-average 200M per day. After checking the relevance with Chinese learning and URL suffixes, the remaining data makes up an on-average $350 \mathrm{~K}$-textfile per day. This remaining data will be used in the following experiments.

\section{B. User Identification}

In this paper, we use users' IP addresses and their identification information to uniquely identify them. Because of the plummeting of the data and the complex conditions required to identify users, our data only provides us close to 14000 different users. Among them, nearly half of the users visit only one page and most of them only issue one session (see Table I). We still have about 7800 users that issued more than one session. These users incited, on average, 2.25 sessions. Most of these sessions are continuous because these users spent more than half an hour on their browsing behaviors.

\section{TABLE I: CHARACTERISTICS OF USERS.}

\begin{tabular}{ll}
\hline Number of users & 14164 \\
Number of users with $>1$ clicks & $7822(55.2245 \%)$ \\
Number of users with $>1$ sessions & $745(5.2598 \%)$ \\
Busiest day in number of users & July 14,2011683 users \\
\hline Average number of query per user & \\
All users & 3.6437 \\
Users with $>1$ queries & 5.7873 \\
\hline Average number of session per user & 1.0671 \\
All users & 2.2497 \\
Users with $>1$ sessions & 1220.7624 \\
\hline $\begin{array}{l}\text { Average visit-time per user } \\
\text { (in second) }\end{array}$ & \\
\hline
\end{tabular}

We extract the IP addresses of these users and locate them according to the location database which is issued by IPligence Lite ${ }^{2}$. After that we propose several analysis of the temporal and geographical distribution of these users.

\section{RESULTS AND DISCUSSIONS}

\section{A. Users' Temporal Distribution}

Fig. 2 shows the temporal distribution of users. There is an obvious cyclic pattern of the distribution (indicated by the green line). According to the Fig, in a specific week, the largest user number always appears on Thursday and among the other six days, Monday also sees a relatively more number of visitors. We have discussed above that, according to statistics, a significant percentage of users visit only one page of this website. Several reasons contribute to this phenomenon, including that this group of users fail to find their expected resources on the homepage or users visit the website by accident. So users who access more than one page provide us with more useful information of the users' distribution because they may be more concerned about

\footnotetext{
${ }^{1}$ The defined suffix set: gif ,jpg , png , bmp , mp3 , doc , docx , xls , ppt , txt , rar, dll, wma, zip, css, js, swf, exe, rm, rmvb, avi , and JPG .

${ }^{2}$ http://www.ipligence.com/free-ip-database
}

Chinese learning compared to other users. Therefore, we extract these users who click more than one page out of the user set and then analyze the change of user number over time (indicated by the purple line). Reader may notice that the fluctuation of the purple line is not as significant as that of the green line, but some cycles still can be observed. Another interesting finding is that there are more one-click users on Thursdays compared to the other days in a specific week according to an observation that the biggest gaps between the green and purple lines usually emerge on Thursday.

The temporal distribution of users whose IP addresses belong to countries and regions outside China shows another interesting characteristic. It is obvious that every Thursday has the biggest number of users. When dividing the two-months period into seven sections according to weekdays (such as Monday, Tuesday and Friday), there is a significant downward trend of accumulated user numbers within these seven sections except the section corresponding to Thursday (see Fig. 1). This downward trend, which indicates that there are more users visiting our Chinese learning website at the beginning of a week rather than visiting at weekends, reveals that people tend to perform higher enthusiasm to learn Chinese on the first few days of a week.

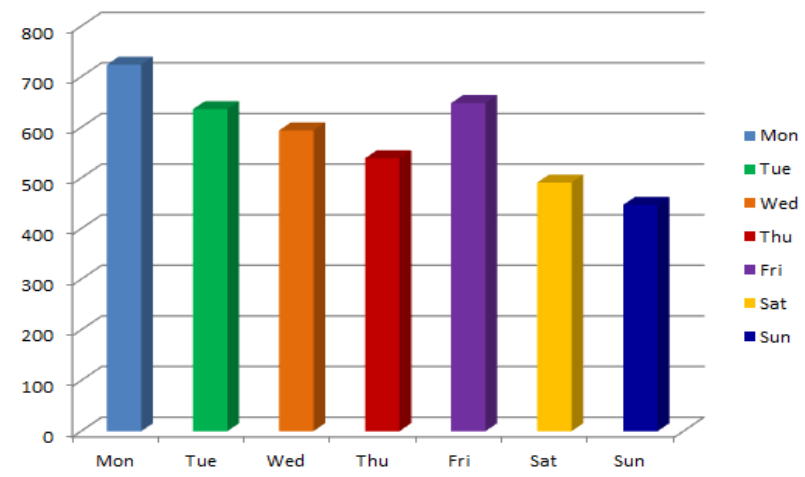

Fig. 1. User distribution over weekdays. In this plot, the $\mathrm{x}$-axis indicates a specific weekday (Monday, Tuesday etc.), the y-axis indicates the total number of users who visited BLCU's website on this specific weekday during July 2011 and August 2011.

\section{B. Users' Geographical Distribution}

Over the two-month period, there are 14164 users in total, of whom nearly $75 \%$ are from Chinese mainland (see Fig. 3). To get a deeper sense of the distribution of foreign users, we treat the foreign users set as a whole and then analyze the composition of this set. The result shows that the United States, Korea and Niue are the Top 3 users' sources. Each of these three countries accounts for over $10 \%$ of the foreign users set. Japan, HongKong, Thailand, Australia, Germany, France and Russia constitute the second echelon of the users' source countries or regions. Each of these countries or regions occupies over $1 \%$ of the foreign users set. There are another 85 countries or regions which make up the other $28 \%$ of the foreign users set (see Fig. 3).

Let us go back to the whole user set including both users whose IP addresses belong to Chinese mainland and those with IP addresses belonging to regions outside China and then sort these source countries and regions. According to the user percentage accounted by these countries and regions, we 
propose eight categories for them and mark them in eight different colors (see Fig. 4). There is no doubt that China (indicated by red) makes the biggest contribution to the user set for its accounting for $74 \%$ of the users. Three countries and regions in total make up the second category (indicated by yellow). They are the United States, South Korea and Niue, which respectively accounts for more than $2 \%$ of the users. Niue is a small country with a population of 1,400 . The reason for its emerging on the top list is that it provides the Internet access service, which means many users absent from Niue can use IP addresses belonging to Niue to get access to the Internet. Japan occupies the third category (indicated by violet). There are two countries in the fourth category (indicated by orange), Thailand and Australia, respectively accounting for over $0.5 \%$ of the user set. Germany, France and Russia consist of the fifth category (indicated by pink).

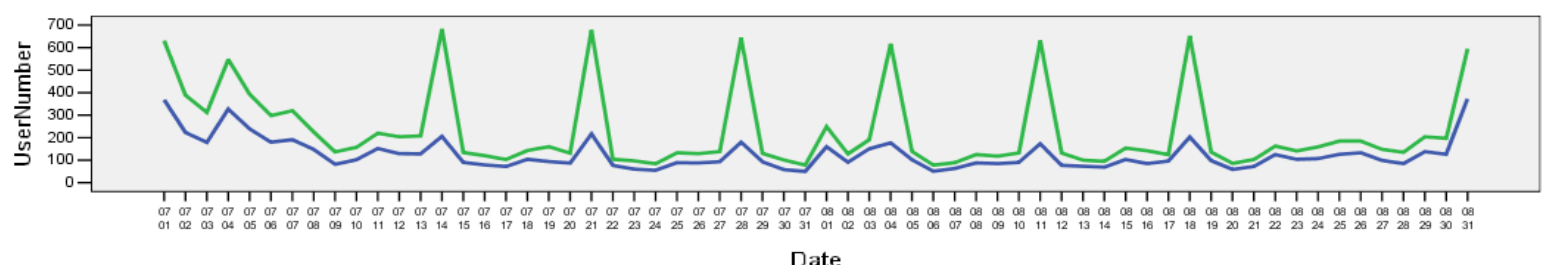

Fig. 2. Distribution of users over time. In this plot, the $x$-axis indicates a specific day, the $y$-axis indicates the user number corresponds to this specific day. The green line indicates the user number's change of all users; the purple line indicates the user number's change of users who click more than one page.
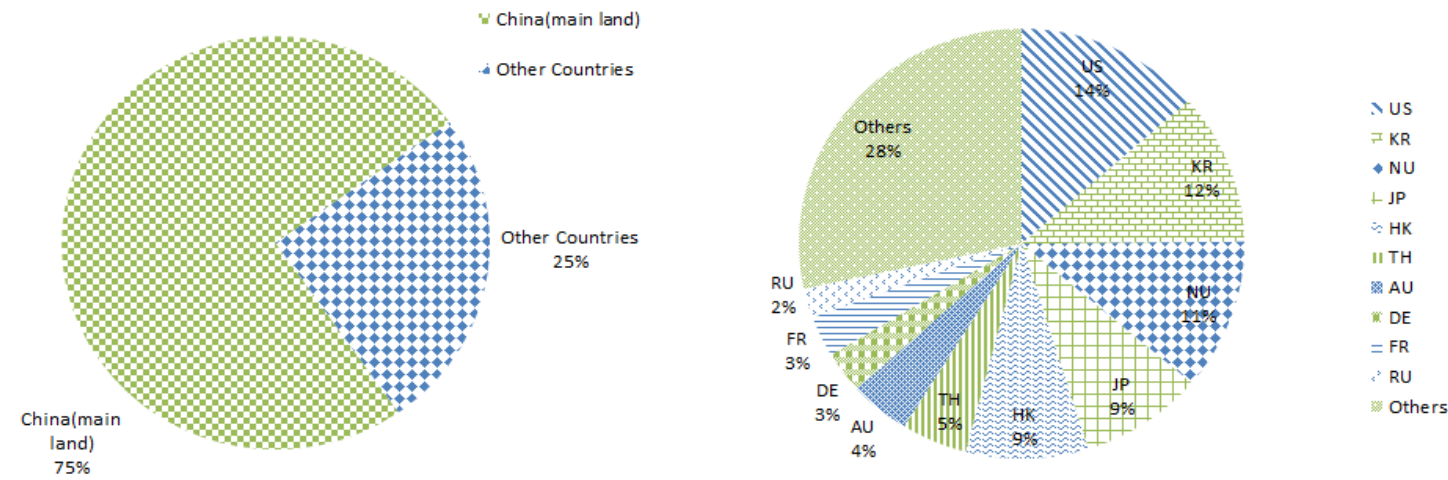

Fig. 3. Pie graph of the website users' geographical distribution. (Left :) The distribution of users whose IP addresses belong to Chinese mainland and users whose IP belong to other countries or regions. (Right :) The geographical distribution of users whose IP addresses belong to foreign countries or regions. Each part of the pie graph indicates the user percentage accounted by the specific area.

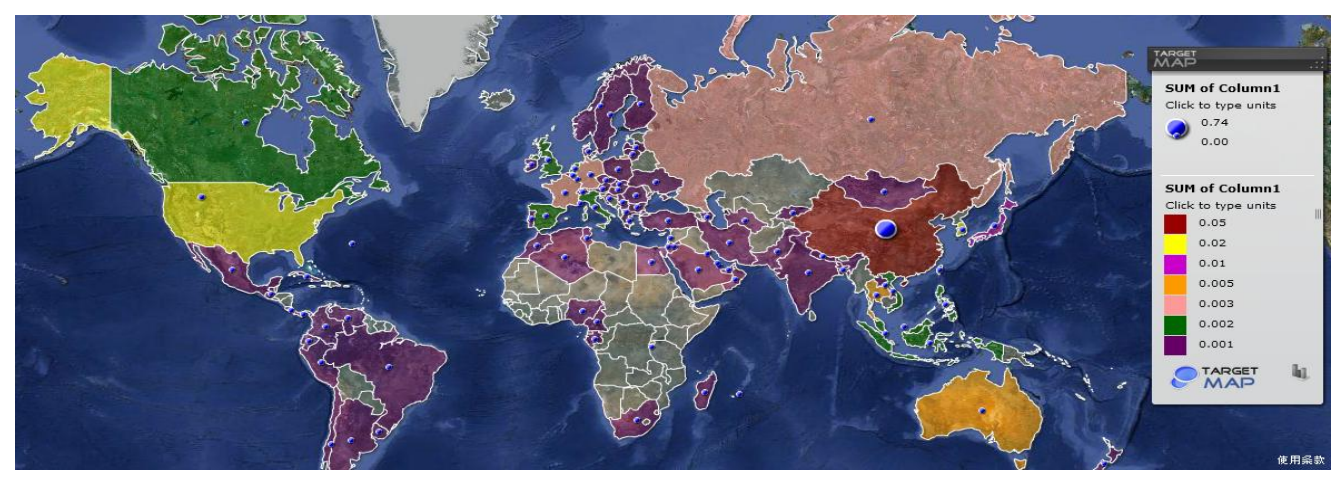

Fig. 4. Classification of countries and regions according to the user percentage accounted by them. Each color patch at the right margin of the Fig indicates a specific class and all the countries and regions belong to this class are marked by the same color. The lower bounds of classes are noted after the color patches.

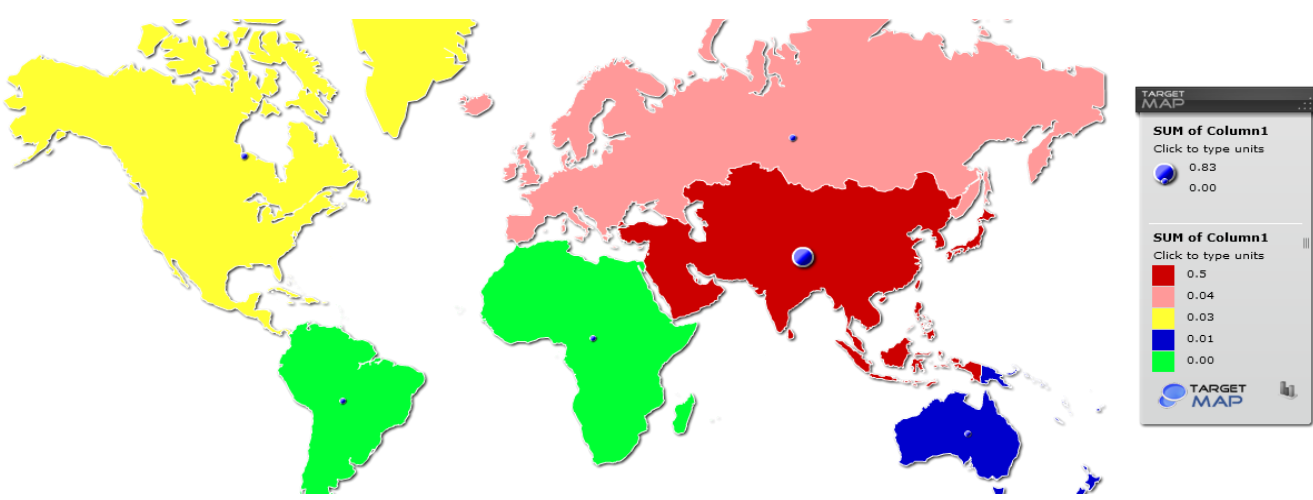

Fig. 5. Classification of continents according to the user percentage accounted by them. Each color patch at the right margin of the Fig indicates a specific class and all continents belong to this class are marked by the same color. The lower bounds of classes are noted after the color patches. 
This data is basically in line with the Chinese Ministry of Education's statistics on the number of foreign students studying in China in 2011. In their statistics, the top ten source countries of foreign students studying in China are the South Korea, Japan, Thailand, Vietnam, Russia, Indonesia, Pakistan and Kazakhstan. In addition, France, Mongolia and Germany also hold their number of foreign students over 5000. The Internet access and the methods of identifying users take some responsibilities for the nuance between our data and the statistics of the Chinese Ministry of Education.

According to the statistics of Chinese Ministry of Education in 2011, Asia is the largest source of the foreign students with its contribution of $64.21 \%$ of the students, followed by Europe (16.15\%), America (11.05\%), Africa $(7.09 \%)$ and the Oceania (1.5\%). So we rearrange our data on a higher level of the continents (see Fig. 5). Asia is the largest source of our website users (indicated by red), with a percentage of $83 \%$ accounted by it. Europe (indicated by pink) is the second with a percentage of $4 \%$, followed by North America, Oceania, South America and Africa. Changes in the list are to some extent caused by the national discrepancies of richness which may lead to differences in the degree of Internet popularity.

\section{CONCLUSION}

In this paper, we have deeply discussed the visitors' temporal and geographical distribution of a Chinese learning website, with web log data contributed by 14164 users from 96 countries and regions over a two-month period. This is the first time that analysis of temporal and geographical user distribution is performed on a Chinese learning website, with our goal to investigate the distribution of Chinese learners. Our result provides some clues for future research in terms of the improvement of Chinese learning websites and the popularization of Chinese. We focused our analysis on both time and space dimensions. The most interesting findings include (a) nearly half of the users just issued one click (users just clicked on just one page) and one session; (b) there is a cyclic pattern of the temporal distribution of the users with its period of one-week and Thursday has the largest user number within a specific week; (c) the data from our web logs is approximately consistent with the statistics, which are issued by the Chinese Ministry of Education in 2011, about the foreign students who study in China at that time.

Our analysis of the Chinese learning website has revealed many directions for future work, including: (i) improving the organization of Chinese learning websites in order to attain better user experience, (ii) offering recommendation and translation services for Chinese learning websites users; (iii) revising the promotion strategies of Chinese language and culture.

\section{REFERENCES}

[1] R. Cooley, B. Mobasher, and J. Srivastava, "Data preparation for mining world wide web browsing patterns," Journal of Knowledge and Information Systems, pp.5-32, Jan.1999.

[2] D. He and A. Goker, "Detecting session boundaries from web user logs," in Proc. $22^{\text {nd }}$ Annu. Information Retrieval Research Conf., Cambridge, pp. 57-66, 2000.

[3] S. Mahlke, "Factors influencing the experience of website usage," in Proc. ACM Conf. Human Factors in Computer System, New York, pp. 846-847, 2002.

[4] H. Heijden, "Factors influencing the usage of websites: the case of a generic portal in the Netherlands," Information\& Management, pp. 541-549, 2003.

[5] H. Heijden, "Using the technology acceptance model to predict website usage: Extensions and empirical test," Research Memorandum, Vrije Universiteit Amsterdam, Netherlands, 2000.

[6] L. B. Cohen, "A two-tiered model for analyzing library website usage statistics, part 1: Web server logs," Portal: Libraries and the Academy, vol. 3, pp. 315-326, April 2003.

[7] S. Beitzel, E. Jensen, A. Chowdhury, O Frieder, and D Grossman, "Temporal analysis of a very large topically categorized Web query log," Journal of the American Society for Information Science and Technology, vol. 58, pp. 166-178, Jan. 2007.

[8] G. Silverstein, M. Heizinger, H. Marais, and M. Morics, "Analysis of a very large Web search engine query log," ACM SIGIR Forum, vol. 3, pp. 6-12, 1999.

[9] W. Weerkamp, R. Berendsen, B. Kovachev, E. Meij, K. Balog, and M. Rijke, "People searching for people: Analysis of a people search engine log," in Proc. 2011 ACM SIGIR Conf., pp. 45-54, 2011.

[10] J. Lin and M. Smucker, "How do users find things with pubmed: Towards automatic utility evaluation with user simulations," in Proc. 2008 ACM SIGIR Conf., pp. 19-26, 2008.

[11] G. Mishne and M. Rijke, "A study of blog search," in Proc. 2006 ECIR Conf., pp. 289-301, 2006.

[12] J. Kim, H. Feild, and M. Cartright, "Understanding book search behavior on the Web," in Proc. 2012 CIKM Conf., Maui, USA.(to be published), 2012.

[13] M. Friendly (2008). Milestones in the history of thematic cartography, statistical graphics, and data visualization. [Online]. Available: http://www.math.yorku.ca/SCS/Gallery/milestone/milestone.pdf

[14] C. Healey, "Choosing effective colors for data visualization," in Proc. $7^{\text {th }}$ IEEE Visualization Conf., pp. 263, 1996.

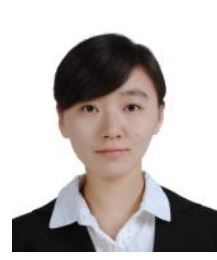

Xiaochen Li will receive her Bachelor degree in information management and information system from Beijing Language and Culture University, Beijing, China in 2013. Her research interests include Web usage, data mining and information retrieval.

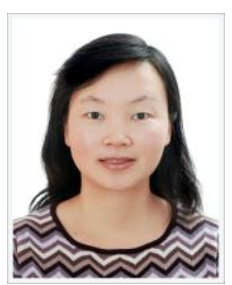

Yan Xu received the M.S. degree in computer science (1996) and the Ph.D. degree in computer science(2004) from Beijing University of Aeronautics \& Astronautics, Beijing, China. She is an Associate Professor in the College of Information Science, Beijing Language and Culture University now. Her research interests include data mining and information retrieval 